\title{
Planetary transit candidates in the CoRoT initial run: resolving their nature ${ }^{\star} \star \star$
}

C. Moutou ${ }^{1}$, F. Pont $^{2}$, F. Bouchy ${ }^{3}$, M. Deleuil ${ }^{1}$, J. M. Almenara ${ }^{4}$, R. Alonso ${ }^{1}$, M. Barbieri ${ }^{1}$, H. Bruntt ${ }^{5}$, H. J. Deeg, ${ }^{4}$, M. Fridlund, ${ }^{6}$, D. Gandolfi ${ }^{7}$, M. Gillon ${ }^{8}$, E. Guenther ${ }^{7}$, A. Hatzes ${ }^{7}$, G. Hébrard ${ }^{3}$, B. Loeillet $^{1}$, M. Mayor ${ }^{8}$, T. Mazeh ${ }^{9}$, D. Queloz ${ }^{8}$, M. Rabus ${ }^{4}$, D. Rouan ${ }^{5}$, A. Shporer ${ }^{9}$, S. Udry ${ }^{8}$, S. Aigrain ${ }^{2}$, M. Auvergne ${ }^{5}$, A. Baglin ${ }^{5}$, P. Barge ${ }^{1}$, W. Benz ${ }^{10}$, P. Bordé ${ }^{11}$, S. Carpano ${ }^{6}$, R. De la Reza ${ }^{12}$, R. Dvorak ${ }^{13}$, A. Erikson ${ }^{14}$, P. Gondoin ${ }^{6}$, T. Guillot ${ }^{15}$, L. Jorda ${ }^{1}$, P. Kabath ${ }^{14}$, H. Lammer ${ }^{16}$, A. Léger ${ }^{11}$, A. Llebaria ${ }^{1}$, C. Lovis ${ }^{8}$, P. Magain ${ }^{17}$, M. Ollivier ${ }^{11}$, M. Pätzold, ${ }^{18}$, F. Pepe ${ }^{8}$, H. Rauer ${ }^{14}$, J. Schneider ${ }^{19}$, and G. Wuchterl ${ }^{7}$

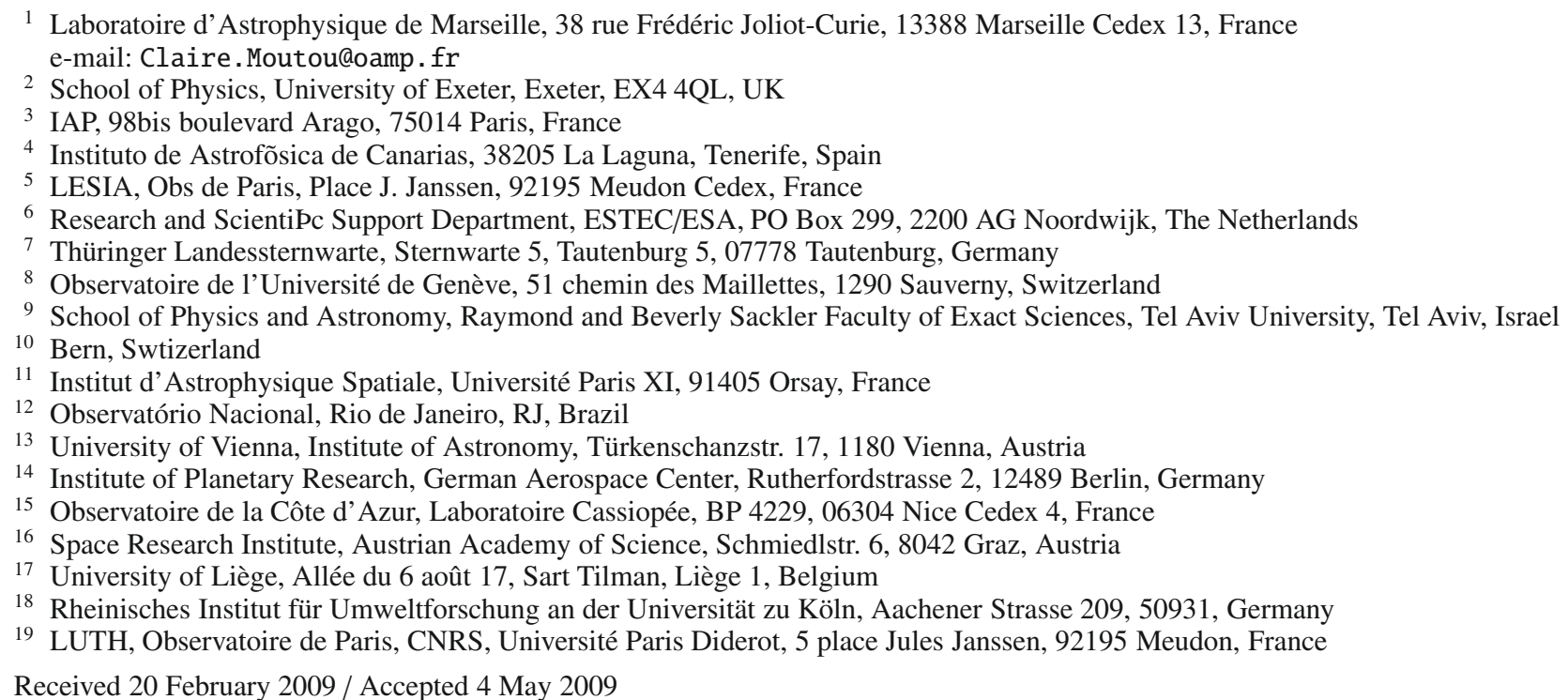

ABSTRACT

With the release of CoRoT lightcurves of the Initial Run IRa01, 50 transiting planetary candidates have been published in a companion paper. About twenty of them were identified as binary stars from the CoRoT lightcurve itself. Complementary observations were conducted for 29 candidates, including ground-based photometry and radial-velocity measurements. Two giant planets were identified and fully characterized. Nineteen binaries are recognized, from which 10 are background eclipsing binaries in the CoRoT mask or triple systems, diluted by the main CoRoT target. Eight cases remain of unclear origin, one of them still being a planetary candidate. Comparison with simulations shows that the actual threshold of confirmed planet detection in this field does not yet fulfill the expectations, and a number of reasons are invoked, like the ranking process based on lightcurve analyses, and the strategy and limits of follow-up observations for targets fainter than magnitude 15.

Key words. stars: planetary systems - techniques: photometric - techniques: radial velocities - techniques: spectroscopic

\section{Introduction}

Compared to ground-based transit surveys, space-based stellar photometry with CoRoT allows the detection of new classes

\footnotetext{
* Based on data obtained at Observatoire de Haute Provence with SOPHIE and with HARPS on the ESO $3.6 \mathrm{~m}$ telescope at La Silla Observatory. The CoRoT space mission, launched on December 27th 2006, has been developed and is operated by CNES, with the contribution of Austria, Belgium, Brazil , ESA (RSSD and Science Programme), Germany and Spain.

* Tables 2 to 13,15 to 17 and Figs. 4 to 7 are only available in electronic form at http://www . aanda.org
}

of transiting planets, characterized by a small radius or a long orbital period. This, however, requires an extensive effort of complementary observations, consisting of ground-based photometry, spectroscopy, and high-precision radial-velocity measurements. This paper presents the analysis of transit candidates detected in the Initial Run of CoRoT, IRa01, obtained by the CoRoT exoplanet science team after two years of complementary observations. The Initial Run took place from $\mathrm{JD}=2454138$ to 2454193 (6 February to 2 April 2007) for a total duration of 55 days. This study is a companion paper to the one presenting the detections themselves (Carpano et al. 2009, hereafter Paper I), with the aim of identifying the best 
scenario and explaining the signal seen by CoRoT, when possible. Paper I presents fifty detected planetary candidates and 141 eclipsing binaries from a total of 9872 targets observed during IRa01. Complementary observations were performed on the 29 most promising planetary candidates. Their folded lightcurve is shown in Figs. 4 to 7 in electronic form.

The instruments and methods used for the complementary observations are listed in Table 1, including the CCD imaging cameras, and spectrographs. Pre-launch observations of the IRa01 targets were performed in 2002-2005, aiming at measuring the magnitudes, colors and positions prior to the launch building the exoplanet entry catalog EXODAT (Deleuil et al. 2009). This information is reported in Table 2. First estimates on the spectral classification of the parent stars were obtained by isochrone fitting of multi-color optical and near-infrared photometry (Barbieri et al., in prep.). After the detection of transit candidates, a classical strategy of follow-up observations was applied, in order to check whether the main target is responsible for the CoRoT transit -or rather, some nearby star included in the photometric mask integrated onboard-, and if the mass ratio between the parent star and the transiting body is small enough to exclude an eclipsing binary. Most difficult to reject are the triple systems or diluted eclipsing binaries within a typical one arcsec radius. For a description of scenarios that mimic a planetary transit and conventional strategies to discard them, see for instance Brown (2003) and Bouchy et al. (2009).

In the following, we will describe case by case all followup observations realized in the context of the IRa01 transiting candidate identification. Not all events described in Paper I required complementary observations. For 20 of them, a binary origin was proposed from the lightcurve analysis itself, or the candidates were not convincing enough. We refer to Paper I for all transit parameters. CoRoT targets and transit candidates are identified with a unique ID (e.g. 0102723949) and a run ID (e.g. IRa01-E1-2046). We will use both identifiers in the tables, figures and section names, but only the shortcut of the run ID (e.g. E1-2046) in the text. CoRoT data of IRa01 are accessible at the URL: http: //idc-corotn2-public.ias.u-psud. fr/ and EXODAT stellar data are available at: http://lamwws . oamp. fr/exodatpub

\section{Resolution of transit candidates}

\subsection{Planets}

\section{E2-1126/0102890318/CoRoT-1b}

The transits of E2-1126 were detected by the alarm mode (Surace et al. 2008), during the CoRoT operations on the initial run. The observations conducted on SOPHIE quickly established the planetary nature of the transiting body, that was then called CoRoT-1b (Barge et al. 2008). The transiting planet is a giant gaseous planet with a $1.49 R_{\text {Jup }}$ radius. The radial-velocity (RV) observations were continued 6 months after this early discovery. We have now gathered 52 measurements with both SOPHIE and HARPS spectrographs, which show no significant variation compared to earlier analyses, neither in terms of long-term drift nor non-zero eccentricity (Pont et al., in prep.). In addition, the transit has been observed by HARPS, and by VLT/FORS2 in $B$ and $V$ filters, to constrain its shape. A better characterisation of the stellar parameters is ongoing from the sequence of HARPS spectra, and especially, the very low metallicity of the parent star is under revision. In-depth analyses of new complementary data on CoRoT-1 will be published in forthcoming papers
(Pont et al., in prep.; Deleuil et al., in prep.; Gillon et al., 2009), while some recent analyses of the CoRoT-1 lightcurve have been published (Bean 2009; Snellen et al. 2009; Alonso et al. 2009).

\section{E1-0330/0102912369/CoRoT-4b}

The candidate E1-0330, with $1.2 \%$ deep transits and a 9.202 day period, has been established as of planetary origin by SOPHIE in October 2007. The data are presented and discussed by Aigrain et al. (2008) and Moutou et al. (2008). The planet has a mass of $0.72 M_{\text {Jup }}$ and a radius of $1.19 R_{\text {Jup }}$, and orbits an F0V star with a rotation period similar to the giant planet's orbital period. Six new HARPS measurements were obtained one year after the earlier announcement. These new data do not change the orbital solution nor indicate a second planet in the system.

\subsection{Eclipsing binaries as the main target}

\section{E1-2046/102723949}

The candidate E1-2046 shows a $1.17 \%$ deep single transit in IRa01 at JD $=2454167.91$. Given the total duration of the time series ( 55 days), this implies that the orbital period is larger than 33 days. It turns out that the same star was also observed during the CoRoT run LRa01, one year later, and that its period is 50.2 days. One SOPHIE spectrum was acquired on 29 February 2008. The cross-correlation function indicates a spectroscopic binary of type 2 (SB2), with two components separated by $31 \mathrm{~km} \mathrm{~s}^{-1}$ (Fig. 1). One exposure was thus sufficient to discard this single-transit candidate as an eclipsing binary. We did not try to constrain the secondary mass with a second measurement, nor to check whether the velocity variation was in phase with the CoRoT ephemeris.

\section{E2-3010/102800106}

CoRoT measured a long period (23.2 days), deep (1.72\%) transit for E2-3010. We performed one HARPS observation on 13 January 2008, at phase $=0.81$. We obtained the cross-correlation function of an SB2 system. The two peak velocities are separated by $23.2 \mathrm{~km} \mathrm{~s}^{-1}$ (Fig. 1). One component is wide $\left(22 \mathrm{~km} \mathrm{~s}^{-1}\right)$ and one is narrow $\left(6.6 \mathrm{~km} \mathrm{~s}^{-1}\right)$, implying different projected rotational velocities. Although we cannot guarantee that there is no planet in this system, confirming it and characterizing its mass would require too large an effort in RV observations. We therefore reject such systems.

\section{E2-3854/102841669}

With a very short period (1.14 day) and a very shallow $(0.136 \%)$ transit signal, the CoRoT lightcurve of candidat E2-3854 seemed very promising. Two SOPHIE observations were made on 28 January and 8 February 2008 (Table 3 ). The cross-correlation function shows two peaks separated by about $80 \mathrm{~km} \mathrm{~s}^{-1}$, which allows us to reject this candidate as an eclipsing binary. With two exposures and the CoRoT ephemeris, we are able to estimate the orbital solution for this system, assuming circular orbits (Fig. 2). The mass ratio of the system is about 1 . Although the primary star is classified as a giant star by pre-launch analysis, the short orbital period refutes this possibility, as the orbital distance of the secondary would have to be smaller than the primary radius; this illustrates how photometric classification can lead to incorrect estimates (see Sect. 3.1). 
Table 1. Instruments and methods used in the complementary observations of the IRa01 candidates.

\begin{tabular}{|c|c|c|c|c|}
\hline Instrument & $\overline{\text { Diam Tel }}$ & Nb Nights $^{a}$ & Method & Measurement $^{b}$ \\
\hline INT/WFC & 2.5 & 5 & photometry & pre-launch spectral typing, astrometry \\
\hline IAC80 & 0.8 & 0.5 & photometry & on-target transit confirmation \\
\hline EulerCam & 1.2 & 3 & photometry & on-target transit confirmation \\
\hline Wise/0.46 & 0.46 & 3 & photometry & on-target transit confirmation \\
\hline Wise/1.0 & 1.0 & 3 & photometry & on-target transit confirmation \\
\hline CFHT/Megacam & 3.6 & 1.5 & photometry & on-target transit confirmation \\
\hline CORALIE & 1.2 & 1 & radial velocity & SB identification \\
\hline SOPHIE & 1.93 & 7 & radial velocity & $\begin{array}{l}\text { SB identification, } \\
\text { mass characterization }\end{array}$ \\
\hline HARPS & 3.6 & 5 & radial velocity & $\begin{array}{l}\text { SB identification, stellar parameters } \\
\text { mass characterization }\end{array}$ \\
\hline UVES & 8.0 & 0.5 & spectroscopy & stellar parameters \\
\hline
\end{tabular}

${ }^{a}$ The approximate number of nights per instrument is given as an indication of the observational effort needed for the follow-up of a single CoRoT field. ${ }^{b}$ SB stands for spectroscopic binary.
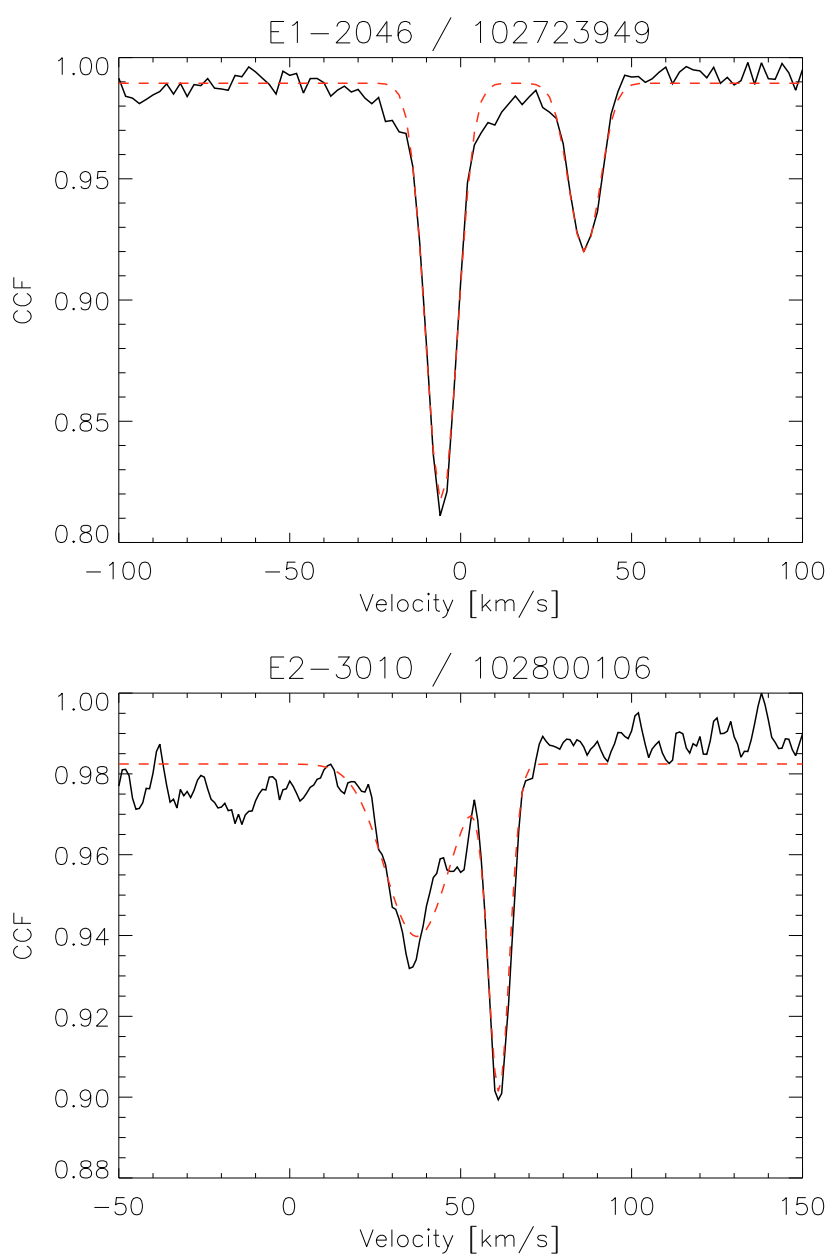

Fig. 1. Cross-correlation functions of two SB2 in the sample, E1-2046 and E2-3010. Dotted lines are Gaussian fits to the data.

\section{E1-1158/102763847}

We followed the candidate E1-1158 (transit depth $1.36 \%$ and orbital period 10.53 days), with the spectrograph SOPHIE on 24 and 29 February 2008 (Table 4). The star is a fast rotator, with $v \sin i \simeq 18 \mathrm{~km} \mathrm{~s}^{-1}$. Photometry indicates an F-type star with $1.5 M_{\odot}$. The velocities obtained near the expected extrema are separated by $46 \mathrm{~km} \mathrm{~s}^{-1}$, hence the system is a spectroscopic
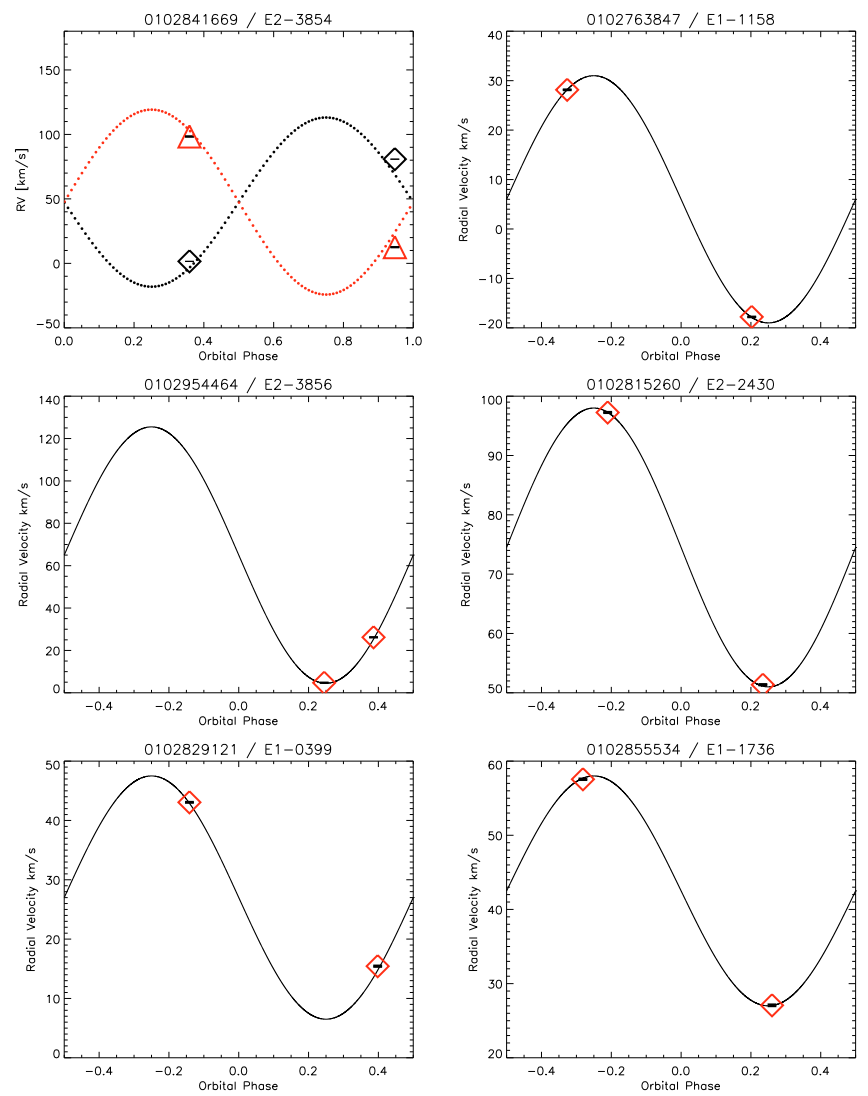

Fig. 2. Radial-velocity curve and rough adjustement of an orbital solution, for one SB2 and 5 SB1 found in our sample.

binary of type 1 (SB1). The semi-amplitude of the orbit, if circular, is $25 \mathrm{~km} \mathrm{~s}^{-1}$, and the mass ratio is 0.22 (Fig. 2).

\section{E1-0399/102829121}

The lightcurve of the candidate E1-0399 shows a 1.5\% deep transit every 33.06 days. The target was observed by CORALIE on 12 and 30 January 2008. The velocities measured at phases 0.86 and 0.39 are separated by $27 \mathrm{~km} \mathrm{~s}^{-1}$, indicating an SB1 system. When the data are fitted with a circular orbit, one gets a semi-amplitude of $20.5 \mathrm{~km} \mathrm{~s}^{-1}$ and a mass ratio of 0.31 . 


\section{E2-3856/102954464}

Despite the long orbital period (16.56 days), the star was observed with the Swiss $1.2 \mathrm{~m}$ Euler telescope at La Silla for on-off photometry. During an on-transit night in April 2008, an eclipse of about $2 \%$ depth was detected on the main target, at the CoRoT predicted ephemeris. The depth is also comparable to the one measured by CoRoT. The star was also observed by HARPS, on 5 March and 24 March 2008. The RV measurements show a single cross-correlation peak, separated by more than $20 \mathrm{~km} \mathrm{~s}^{-1}$ from the first night to the second (Table 6), while only sampling a small fraction of the orbit. Under the assumptions that the spectroscopic binary follows the CoRoT ephemeris (since it is transiting), and that the orbit is circular, it would correspond to a semi-amplitude $\mathrm{K}$ of about $60 \mathrm{~km} \mathrm{~s}^{-1}$ (Fig. 6). The mass ratio between both components of the stellar system would then be 0.7 .

\section{E2-0203/0102825481}

The transit candidate E2-0203 was identified by the alarm mode, in March 2007, when the CoRoT observations of the IRa01 field were still ongoing. The transits are of $3.4 \%$ depth and with a period of 5.168 days. Ground-based photometry with the IAC $80 \mathrm{~cm}$ (30 March 2007) telescope and Canadia-France-Hawaii telescope (10 APril 2007) confirmed the eclipse occuring on the main star of the CoRoT photometric aperture. The spectroscopic observations started on 26 March 2007 at Tautenburg observatory, and were continued until 18 April. The star is a spectroscopic binary at the period and phase determined by CoRoT. The RV semi-amplitude inferred from the observations is $16.9 \mathrm{~km} \mathrm{~s}^{-1}$. The primary star is of slightly later type than the Sun, and the secondary is a low-mass $M$ star. With a mass ratio of 0.142 and radius ratio 0.184 , this secondary belongs to the rare population of very low-mass stars. The refined analysis of the complete lightcurve shows the detection of the secondary eclipse, 40 times shallower than the primary transit; it is detected with $11 \sigma$ significance at phase 0.514 , as expected for a slightly eccentric orbit (Fig. 4). An in-depth analysis of E2-0203, including a discussion of the secondary transit, the orbital eccentricity, and additional measurements, will be presented in an article by Morales et al. (in prep.).

\section{E2-2430/102815260}

CoRoT observations of E2-2430 have revealed a transit event of $1.16 \%$ depth and 3.587 day orbital period. SOPHIE observations of E2-2430 were conducted on February 5 and 7, 2008, near the expected RV extrema (Table 7). The measurements are separated by $46 \mathrm{~km} \mathrm{~s}^{-1}$. The parent star is a fast-rotating F-type star (projected velocity of $30 \mathrm{~km} \mathrm{~s}^{-1}$ ), indicated by a wide cross-correlation function and few absorption lines in the optical spectrum. This system is an SB1, with a semi-amplitude of $23.5 \mathrm{~km} \mathrm{~s}^{-1}$ and mass ratio of about 0.17 (Fig. 2).

\section{E2-1736/102855534}

We acquired two SOPHIE observations of E2-1736 (1.18\% deep transit at period 21.72 days) on 31 January and 10 February 2008 (Table 8). The star is rotating rapidly, with a $v \sin i$ of $27 \mathrm{~km} \mathrm{~s}^{-1}$, and the velocity difference is about $30 \mathrm{~km} \mathrm{~s}^{-1}$ between both expected extrema (Fig. 2). If the orbit is circular, which is questionable at the $21 \mathrm{~d}$ period, then the RV semi-amplitude would be $15.5 \mathrm{~km} \mathrm{~s}^{-1}$, corresponding to a mass ratio of 0.2 . Further analysis of the CoRoT lightcurve showed a faint secondary detected at 9- $\sigma$ (Fig. 4).

Nine spectroscopic binaries were thus identified among the IRa01 transit candidates. It is noticeable that most of them have relatively low amplitudes of velocity variations, corresponding to small mass ratios, ranging from 0.14 to 0.3 . This is explained by the fact that other types of binaries are easily rejected from the lightcurve analysis itself (Paper I). Only for extreme mass ratios is the secondary transit undetected in the high-precision and continuous CoRoT lightcurves. Along the same line, most of these binaries have long orbital periods, i.e. more than 10 days, for which no strong ellipsoidal variation is expected to appear in the lightcurve. This was expected from pre-launch simulations and is fully validated by the analysis of IRa01 candidates.

\subsection{Background eclipsing binaries}

The first cases presented in this section have been solved by photometry; they correspond to background eclipsing binaries (BEB) hidden within the wide photometric mask of CoRoT (typical size 20 arcsec). The other cases have been solved by spectroscopic observations. They usually correspond to blends with eclipsing binaries typically within the limits of ground-based seeing, ie 1 arcsec. Some have both photometric and RV evidence, allowing us to precisely describe the scenario, which is very likely related to physically associated triple systems.

\section{E2-2604/102805893}

Ground-based photometry with Euler in April 2008 was performed on this shallow $(0.25 \%)$ transit, short period (3.82 days) candidate. On-off relative photometry showed no transit occurring on the main target. A nearby star, however, undergoes a $3 \%$ eclipse. The star is distant by 15 arcsec and 7.5 times brighter in $\mathrm{R}$ than the main target. With the known CoRoT PSF and the photometric aperture used, we estimated that about $1 \%$ of the flux of this background star pollutes the CoRoT mask (including distance and flux difference). Thus, the transit observed by CoRoT is caused by this background eclipsing binary.

\section{E2-1136/102809071}

The candidate E2-1136 also shows a short period transit signal with very shallow depth $(0.17 \%$ and 1.224 day, respectively). On-off photometry performed both at Wise Observatory and with Euler found a nearby star with a $8 \%$ deep eclipse. The transit occurs on a stellar contaminant at 12 arcsec towards the east. Dilution from that star may explain the observed $0.17 \%$ deep Corot lightcurve. A deep analysis of the CoRoT lightcurve -not available at the time of follow-up operations- shows a secondary at opposite phase detected at a 8- $\sigma$ level, further confirming the binary scenario (Fig. 4).

\section{E2-4300/102802430}

The lightcurve of E2-4300 shows a $0.5 \%$ deep transit occuring every 5.806 days. It was observed at a predicted transit time at Canada-France-Hawaii Telescope with Megacam on 10 January 2008. A nearby star at 10 arcsec towards the south-east and 0.95 mag fainter undergoes a $7 \%$ eclipse, at the expected epoch, while the main target has no photometric variation. The CoRoT transit is clearly caused by a BEB in the photometric mask. 


\section{E2-3425/102835817}

The lightcurve of E2-3425 shows transits of $0.36 \%$ depth and 1.185 day period. The star was observed with HARPS in the high efficiency mode EGGS on 5 and 7 October 2008. The typical uncertainty on such a faint star with EGGS is of the order of $30 \mathrm{~m} / \mathrm{s}$. No variation is observed at this level between both measurements taken close to the expected RV extrema (Table 16). Ground-based photometry was performed on 31 January 2009 with Euler for this target, and shows that a star 1.2 times fainter than the main target and distant by 12 arcsec undergoes a $25 \%$ deep eclipse approximately at the predicted epoch. A shift of the central transit time of half an hour is observed, that is compatible with the measured ephemeris error and the gap between the CoRoT lightcurve and the Euler observation.

\section{E1-0288/102787048}

CoRoT detects a $0.37 \%$ deep transit with a 7.89 day orbital period in the lightcurve of E1-0288. Follow-up observations of E1-0288 started with ground-based photometry at the Observatoire de Haute Provence with the $120 \mathrm{~cm}$ telescope. Aperture photometry was performed on the seeing-limited images, and the conclusion was consistent with a transit occuring on the target star, at the expected CoRoT ephemeris. Radial velocity observations then started with SOPHIE; three measurements were done, from which no conclusions could be drawn (no RV variation). A second look at the OHP $120 \mathrm{~cm}$ data with DECPHOT (Gillon et al. 2007) then allowed us to separate the main target from a nearby star about 9 arcsec north and 1.3 magnitude fainter. When both stars can be separated, a 3\% deep eclipse is detected on the faintest one. Once diluted in the flux of the main target, it well reproduces a $0.35 \%$ deep transit as seen by CoRoT. In parallel, we performed two measurements with HARPS on the nearby contaminant, and detected an SB1 with a semi-amplitude of $14.5 \mathrm{~km} \mathrm{~s}^{-1}$ (Bouchy et al. 2009), hence a mass ratio of 0.15 . This is another low-mass stellar companion, with characteristics similar to E2-0203, as well as a BEB as suggested by the signal detected by CoRoT.

\section{E2-0704/102855472}

On-off photometry was performed with Euler on candidate E2-0704. However, these measurements were not obtained at an optimal orbital phase, and therefore the result is not conclusive. A nearby star located 11 arcsec to the south and five time fainter than the main target is a potential background eclipsing binary. HARPS observations obtained on 29 February and 1 March 2008 show no velocity variations between extreme phases (Table 9). It thus suggests that this eclipsing binary is in the background, within the CoRoT photometric mask, rather than the main target, although we do not have the ground-based confirmation of this scenario. Moreover, the secondary eclipse is unambiguously detected by CoRoT at the expected phase, and spectroscopic analysis of HARPS data shows that $T_{\text {eff }} \simeq 5400 \pm 200 \mathrm{~K}$ and $\log g \simeq 3.0 \pm 0.5$; the main CoRoT target being a giant star, it is unlikely that it has a companion with a 2.15 day period, so the eclipsing binary detected by CoRoT has to be a nearby target.

\section{E2-1857/102798247}

The transit event of E2-1857 has $0.47 \%$ depth and an extremely short period of 0.822 days. SOPHIE observations of E2-1857 started in February 2008. We obtained

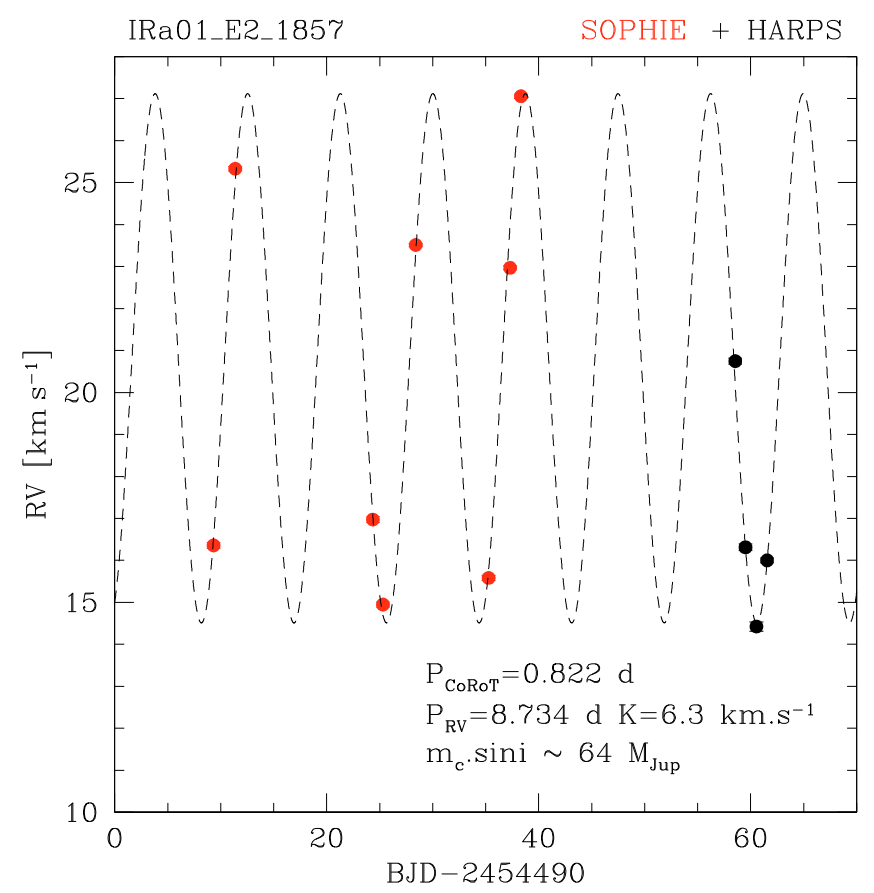

Fig. 3. The radial-velocity variation of E2-1857 as a function of time, including SOPHIE and HARPS variations. The superimposed orbit has a period of 8.734 days whereas the CoRoT period of 0.822 day is caused by a nearby background eclipsing binary.

8 measurements (Table 10), and measured a radial velocity fluctuation of a few $\mathrm{km} \mathrm{s}^{-1}$. A few measurements were also taken with HARPS in order to observe the bisector behaviour. With this data set, we found an orbital solution at a period of 8.734 days, whereas the CoRoT period is 0.822 day (Fig. 3). In parallel, on-off observations took place on Euler on 05 February 2008. After deconvolution photometry, an eclipsing nearby star is detected at 3 arcsec north-west of the main target, in phase with the CoRoT ephemeris. This star is 38 times fainter in $\mathrm{R}$ than the target. It undergoes a $11.5 \%$ drop in brightness during the expected transit while the target is stable. We thus observe both an eclipsing binary (a nearby faint star producing the CoRoT signal, diluted by the main target) and a spectroscopic binary (the main target). The eclipsing binary also shows a secondary eclipse at CoRoT phase 0.5, detected at the 10- $\sigma$ level since then (Fig. 4). The primary target E2-1857 is a dwarf with an estimated effective temperature of $7200 \mathrm{~K}$; its mass may be approximated as 1.6 $M_{\odot}$. The semi-amplitude of the RV variations is $6.3 \mathrm{~km} \mathrm{~s}^{-1}$ at the 8.734 day period, which corresponds to a minimum mass of the companion of $0.1 M_{\odot}$. It is unlikely that two unrelated systems are seen edge-on within 3 arcsec from Earth, so that the real mass is probably significantly higher. The primary star E2-1857 is also classified as a $\delta$ Scuti variable.

\section{E2-2009/102788073}

In the lightcurve of E2-2009, CoRoT detected a transit of $0.41 \%$ depth occuring every 10.85 days. We gathered 7 SOPHIE spectra of E2-2009 between 6 February and 20 March 2008. With uncertainties ranging from 20 to $60 \mathrm{~m} / \mathrm{s}$, we could not detect significant RV variation at the CoRoT ephemeris. On 7 March 2008, Euler on-off photometric observations detected no variations, either on the main target or on the surrounding stars. This result could have indicated a potential small planet scenario, since the 
CoRoT detected transit is rather shallow (0.004). We therefore performed two additional measurements with HARPS, with a greater accuracy than SOPHIE, on 22 and 25 March 2008, at phases 0.50 and 0.77. All RV measurements of E2-2009 are displayed in Table 11 and were presented by Bouchy et al. (2009) (see their Sect. 4.4 and Fig. 3). The difference between the measurements is from about $50 \mathrm{~m} / \mathrm{s}$ to more than $200 \mathrm{~m} / \mathrm{s}$, depending on the numerical template spectrum that is used for the spectral cross-correlation (F0 to K5). Such a significant template dependence of the derived radial velocity is observed for triple systems, or binary systems blended with another stellar source. The largest velocity variation corresponds to the mask with closest spectral type to the primary star of the binary system, in this case, a K-type star. The bisector span also varies with the velocity, another signature of a diluted binary (Bouchy et al. 2009). Now, the RV variation should still be in phase with the CoRoT ephemeris to explain the detected photometric signal, which is not the case. The probable scenario is therefore a binary $\mathrm{K}$ system diluted by the CoRoT main target; having similar colours, it is not possible to distinguish primary and secondary transits of this sytem, while both are detected. The orbital period is then double the one measured by CoRoT, i.e. 21.691 days. Recalculating the phase, we now find a good agreement with the RV variation and the photometric signal (see Table 11). This scenario is also reinforced by 1) the observation of a slight asymmetry in the HARPS crosscorrelation function, and a bisector effect; and 2) a V-shape transit, both findings suggesting a diluted grazing binary.

\section{E1-2329/102869286}

The candidate E1-2329 shows a transit of $0.37 \%$ depth and 1.876 day period in the CoRoT ligthcurve. It was observed with HARPS on 22, 25 and 28 February 2008. The derived velocities are given in Table 12. As for E2-2009, we found a behaviour of the velocity that depends on the mask used for correlating the spectrum. In this case, no variation is observed with masks of K5 and G2 stars, while a $80 \mathrm{~m} / \mathrm{s}$ velocity difference is seen when using the F0 mask (the error of HARPS observations is less than $20 \mathrm{~m} / \mathrm{s}$ ). This would mean an F-type primary of a binary system, blended by the CoRoT main target. The velocity variation is not consistent with the CoRoT ephemeris. If the period was double, however, the velocity would fit the expected behaviour. Thus this system, most probably, is a triple system with two eclipsing stars of the same colour, hotter than the Sun, and with an orbital period of 3.734 days.

\section{E1-3336/102876631}

The CoRoT transits of E1-3336 are $0.17 \%$ in depth, with a period of 1.389 day. Three SOPHIE spectra of E1-3336 were obtained early in February 2008. We could derive a low-amplitude RV variation in phase with CoRoT, and we continued the observation with HARPS to obtain higher accuracy. The 3 additional data points obtained with HARPS showed a different behaviour when using different cross-correlation masks (Table 13 and Fig. 3 in Bouchy et al. 2009). The highest RV amplitude is found for the F0 mask, implying an early-type primary star in a blended binary system. The velocity variation of the blended binary is in phase with the CoRoT ephemeris; the diluted eclipses of this double star are thus the origin of the photometric signal.

\subsection{Unsolved cases}

This section deals with stars for which spectroscopy revealed a fast rotating star or a hot star. Radial velocity measurements are impossible, or very limited in precision.

\section{E1-0783/102895957}

E1-0783 is a target with a single $0.64 \%$ transit in the full 58 day lightcurve, occuring 25 days after the initial epoch. Although the period is not measured, and ground-based photometric confirmation is impossible, the candidate is potentially of great interest for periods longer than 32 days. SOPHIE observed E1-0783 on 26 October 2008 and no cross-correlation function could be detected. The star is of early type with an effective temperature of about $7500 \mathrm{~K}$, and a projected velocity higher than $350 \mathrm{~km} \mathrm{~s}^{-1}$ to blur the lines. Spectroscopy will not provide clues of possible grazing binaries. The transit depth marginally allows a secondary of planetary radius if the transit occurs on the main target: with a stellar radius of 1.4 to $3.0 R_{\odot}$, the transiting body has a radius larger than $1.1 R_{\text {Jup }}$.

\section{E1-4617/102753331}

The transit of E1-4617 is rather deep (4\%) and of long period (19.76 days). In order to confirm or reject the planet hypothesis, HARPS observed this target on 19 February 2008, and could not detect a cross-correlation function. In the spectrum, only hydrogen Balmer lines and interstellar $\mathrm{NaI}$ doublet are visible. The star is of spectral type A, and radial velocity measurements are not possible. With a stellar radius greater than 2 solar radii, the $4 \%$ deep transit is however of stellar origin, rather than planetary. Pre-launch photometry indicates a main-sequence $\mathrm{G}$ star (Table 2), but degeneracy with a reddened early-type star is expected, even if requiring a relatively extreme colour excess of $0.6 \mathrm{mag}$, and we are more confident of spectrosocpy for this rough spectral typing.

\section{E2-4467/102940315}

The 16.45 day period transit candidate E2-4467 (1.4\% depth) was observed with HARPS on 27 February 2008. No crosscorrelation function is detected and the spectrum shows only Balmer lines. This case is similar to E1-4617: the depth of the transit $(1.4 \%)$ and the early-type primary makes a planetary origin impossible for the transit.

\section{E1-4591/102806520}

CoRoT detects $0.29 \%$ deep transits with a period 4.29 days in E1-4591. We obtained one HARPS spectrum of this target on 2 March 2008. With one hour exposure and a signal-to-noise ratio of about 20 at $550 \mathrm{~nm}$, we have detected a very weak and wide cross-correlation function, characterized by a contrast of about $1 \%$ and a width of $21 \mathrm{~km} \mathrm{~s}^{-1}$. This width corresponds to a projected velocity of about $16.4 \mathrm{~km} \mathrm{~s}^{-1}$. The primary star is a modestly rotating $\mathrm{F}$ star. A second measurement was planned but never performed, because of the very low contrast of the cross-correlation function which prevents high-precision velocity measurements. If the rotation period of the star was the same as the orbital period, as in a synchronised binary system, it would lead to a $1.38 R_{\odot}$ primary star, and a secondary of $0.7 R_{\text {Jup }}$. This suggests a companion in the stellar mass range, able to trigger tidal synchronisation. Additionnally, the lightcurve of 
this target shows $\delta$-Scuti-type periodic pulsations at a frequency of 11.8741 cycles/day (Fig. 4); strangely, the transit period is exactly 51 times the pulsation period, but such a large factor could be coincidence, rather than a signature of tidal interactions.

\section{E2-1712/102826302}

The candidate E2-1712 has transits of $0.24 \%$ every 2.767 days. Ground-based photometry of E2-1712 was performed at the Wise Observatory on 28 December 2007. It was found that no nearby target undergoes eclipse, and the transit event detected by CoRoT is not seen either, with only $0.24 \%$ depth. It is thus inferred that the CoRoT transit occurs on the main target and the background eclipsing binary scenario is rejected. Then, the star was observed with HARPS on 27 February and 1 March 2008: the cross-correlation function is $18 \mathrm{~km} \mathrm{~s}^{-1}$ wide, showing a fast rotating star; the projected velocity is $13.4 \mathrm{~km} \mathrm{~s}^{-1}$. The RV variations between both measurements at phases 0.72 and 0.17 is $60 \mathrm{~m} / \mathrm{s}$, but, due to the rotationally broadened spectral lines, individual errors are as large as 59 and $54 \mathrm{~m} / \mathrm{s}$, thus of the order of the observed variation. A possible variation of the stellar line profile is observed, which could indicate a diluted eclipsing binary. Also, a rough determination of stellar parameters from the HARPS spectra shows that the parent star is evolved, with $T_{\text {eff }}=6700 \pm 100 \mathrm{~K}$ and $\log g$ between 3.5 and 4.0. The most probable scenario is a triple system, yet we cannot firmly conclude on this case.

\section{E1-4998/102821773}

The candidate E1-4998 shows a 1.9\% deep transit at period of 10.08 days. We obtained one HARPS measurement of the star on 21 January 2008 and a second point on 27 February 2008 (Table 15). The observed variation is of $23 \mathrm{~km} \mathrm{~s}^{-1}$ but not in phase with the CoRoT ephemeris, nor with twice the CoRoT period. We can suggest a binary at a different period superimposed on a planet-hosting star or another binary. The available data and the low brightness of the star do not allow us a more definite solution. No ground-based photometry has been performed on this target, so that a BEB is not excluded. So this case remains unsolved.

\section{E2-1677/102874481}

E2-1677 shows a single transit of 3\% depth detected in the IRa01, featuring a system with a period longer than 33 days. One spectrum was obtained with SOPHIE on 22 December 2008. It shows a fast rotating star and the cross-correlation function is too noisy for high-precision RV measurements, especially with no constraint on the orbit's ephemeris. On the other hand, photometric colours indicate an F type star; the deep transit then is probably due to a stellar companion rather than of planetary origin, and it could obviously be a spatially resolved BEB in the CoRoT mask as well.

\section{E1-4108/102779966}

Finally, E1-4108 shows periodic $0.5 \%$ transits with a 7.366 day period. Ground-based photometric observations obtained with CFHT/Megacam on 10 March 2008 indicate that the transit occurs on the main CoRoT target. Three HARPS measurements were performed in February 2008, which show no significant $\mathrm{RV}$ variation at the level of $20 \mathrm{~m} / \mathrm{s}$ (Table 17). A preliminary
Table 14. Status of transit candidate resolution following the analysis of complementary observations.

\begin{tabular}{llllll}
\hline \hline WinID & Depth & $\begin{array}{l}\text { CoRoT P }^{a} \\
\%\end{array}$ & $\begin{array}{l}\text { Nature } \\
\text { [d] }\end{array}$ & K & $\left.\begin{array}{l}\text { RV P } \\
{\left[\mathrm{km} \mathrm{s}^{-1}\right]}\end{array}\right]$ \\
\hline E1-2046 & 1.17 & $50.2^{b}$ & SB2 & $>15$ & - \\
E2-3010 & 1.72 & 23.2 & SB2 & $>17$ & - \\
E2-3854 & 0.136 & 1.14 & SB2 & 43 & - \\
E2-3856 & 2.05 & 16.56 & SB1 & 60 & - \\
E1-1158 & 1.36 & 10.53 & SB1 & 25 & - \\
E2-0203 & 3.43 & 5.168 & SB1 & 16.9 & - \\
E2-2430 & 1.16 & 3.587 & SB1 & 23.5 & - \\
E1-0399 & 1.5 & 33.06 & SB1 & 20.5 & - \\
E2-1736 & 1.18 & 21.72 & SB1 & 15.5 & - \\
E2-2604 & 0.25 & 3.82 & aperture BEB & - & - \\
E2-1136 & 0.17 & 1.22 & aperture BEB & - & - \\
E2-4300 & 0.51 & 5.806 & aperture BEB & - & - \\
E1-0288 & 0.37 & 7.89 & aperture BEB & - & - \\
E2-3425 & 0.36 & 1.185 & aperture BEB & - & - \\
E2-1857 & 0.47 & 0.822 & unresolved BEB & 6.3 & 8.734 \\
E2-2009 & 0.41 & 10.85 & unresolved BEB & - & 21.69 \\
E1-2329 & 0.37 & 1.867 & unresolved BEB & - & 3.734 \\
E1-3336 & 0.17 & 1.389 & unresolved BEB & - & - \\
E2-0704 & 0.72 & 2.15 & unresolved BEB & & \\
E1-4617 & 4.0 & 19.76 & hot/fast star & - & - \\
E1-0783 & 0.64 & $>32$ & hot/fast star & - & - \\
E2-4467 & 1.4 & 16.45 & hot/fast star & - & - \\
E1-4591 & 0.29 & 4.295 & hot/fast star & - & - \\
E2-1677 & 3.0 & $>33$ & hot/fast star & & \\
E2-1712 & 0.24 & 2.767 & unknown & - & - \\
E1-4998 & 1.9 & 10.08 & unknown & $>23$ & - \\
E1-4108 & 0.5 & 7.366 & unknown & $<0.02$ & - \\
E2-1126 & 2.16 & 1.509 & planet & 0.188 & - \\
E1-0330 & 1.2 & 9.202 & planet & 0.063 & - \\
\hline
\end{tabular}

${ }^{a}$ for a more accurate determination of CoRoT period, see Paper I. ${ }^{b}$ The period of this candidate was not measured during IRa01, but was inferred after it was re-observed by CoRoT during LRa01 at the end of 2008.

analysis of stellar fundamental parameters in these HARPS spectra shows that it is a cool main-sequence star (Table 2 and Deleuil et al., in prep.), with estimated mass and radius of $0.8 M_{\odot}$ and $0.8 R_{\odot}$. Such a faint star requires a large effort in spectroscopy for a complete confirmation, which has not been achieved so far. Preliminary information points towards a sub-Jovian planetary candidate (less than 3 times Neptune's mass and 1.6 times Neptune's radius), but for which no mass determination has yet been made. Alternatively, it could also be a triple system or diluted EB.

We are left with 8 cases for which no definite solution can be given. Most of them consist of fast rotators or early-type stars, for which no high-precision radial-velocity measurement is possible. E1-4108 is our only planetary candidate left, with a confirmation that the transit occurs on the main target and a mainsequence cool parent star. All the conclusions of our complementary observations, and their analysis, are given in Table 14.

\section{Statistics}

\subsection{Stellar population}

We used pre-launch observations (Deleuil et al. 2009) for a statistical estimate of the stellar population in IRa01. The classification method (Barbieri et al., in prep.) uses a comparison of magnitudes with reddened isochrones of stars of different types. The best-fit solution is extracted using Bayesian statistics. At small 

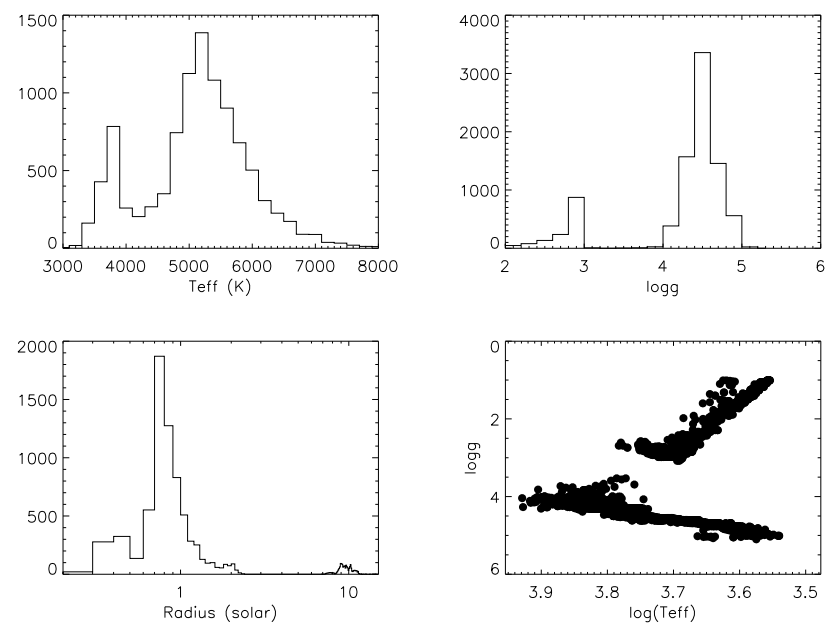

Fig. 8. The histograms of effective temperatures, $\log g$, first-order estimate of the stellar radius in the IRa01 target sample, and HR-diagram equivalent of this stellar field presented as $\log g$ versus effective temperature (bottom right).

latitudes above the Galactic plane, the CoRoT fields are characterised by an inhomogeneous interstellar content, which strongly affects identification of the evolutionary stage of a given target: a nearby red dwarf may be confused with a distant giant, or the opposite, as for candidates E2-2430 and E2-3854. The stellar radius may be strongly misestimated for certain classes of effective temperature, and thus the planet-likelihood ranking of a detected transit event must not rely only on a photometric analysis. It was thus decided for the follow-up campaign of IRa01, not to withdraw an event based on its being estimated as a giant.

The stellar field observed during the IRa01 is located at about RA $=06 \mathrm{~h} 47 \mathrm{mn}$ and Dec. $=-01^{\circ} 51^{\prime}$ or Galactic coordinates $[214.0,-1.9]$. The stellar population of IRa01 targets, after selection in the field, is composed of 9872 stars in the magnitude range $r=11.25-16$. Histograms of parameters issued from pre-launch photometry are shown in Fig. 8, as well as a temperature-luminosity diagram. The population of this target sample is dominated by the main sequence, which comprises up to $75 \%$ of the targets. According to our classification scheme, the mean radius is about $1 R_{\odot}$ and the mean effective temperature is below $6000 \mathrm{~K}$. This is not to be compared directly with models of the Galaxy (Robin et al. 2004), since some selection criteria have been applied. The number of dwarf stars is overestimated by $25 \%$ in our target sample, compared to a simulation with the complete stellar field. This difference is due to a combination of 1) the selection of targets; 2) misclassification of giant stars; and 3) an ill-constrained reddening law in the Galaxy model towards this line of sight.

\subsection{Discussion of statistics}

We recall that 191 transiting candidates have been detected in IRa01, among which 141 are obvious eclipsing binaries, and 29 of the 50 planetary candidates were followed up for confirmation. The results of the present study in terms of transiting planet detection can be compared to expectations, both to assess the efficiency of the survey and to constrain the statistical properties of exoplanets. With only two planets detected in IRa01, however, a very detailed analysis is not warranted at this point. We give here our main conclusions on the topic, and refer further discussion to global analyses including more CoRoT fields.

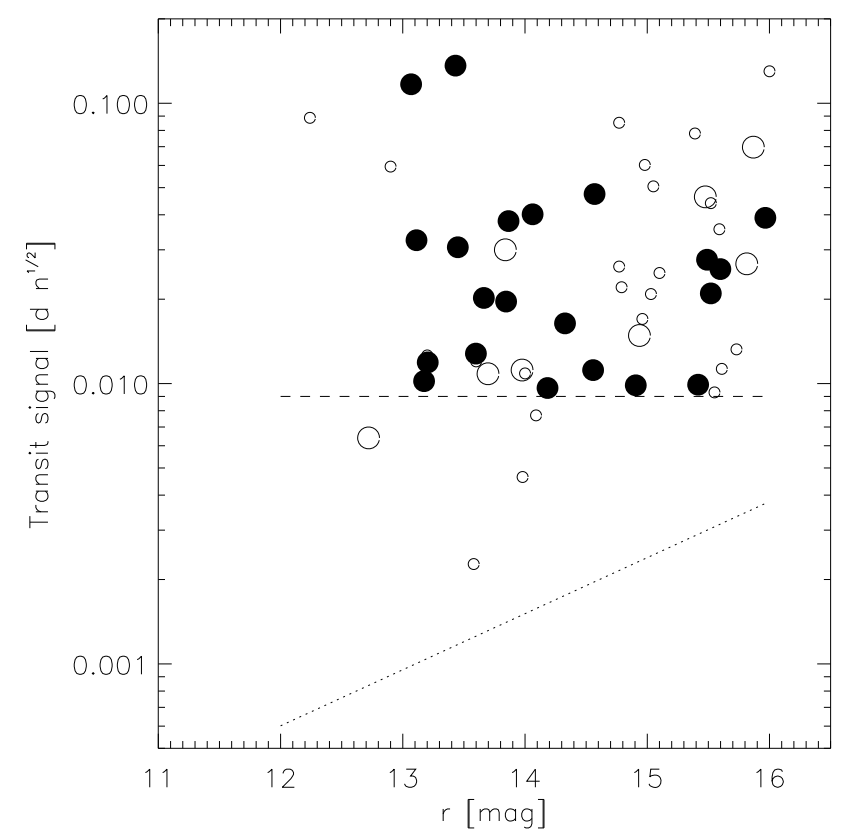

Fig. 9. Transit signal vs. magnitude for the planetary transit candidates discussed in this paper (large symbols). Closed symbols are objects confirmed as eclipsing binaries or transiting planets. Small symbols show the location of candidates of Paper I that were not followed up. The dashed line shows the threshold model, the dotted line shows the threshold based on ideal assumptions.

\section{Transit detection threshold}

We model the transit signal detection threshold as in Pont et al. (2006). That study shows that a threshold model as a signalto-noise cutoff $\frac{d \cdot n^{1 / 2}}{\sigma^{1 / 2}}>S_{T}$ provided a good description for magnitude-limited photometric transit surveys included in this study as a function of magnitude and transit signal $\left(d \cdot n^{1 / 2}>3\right.$, $\sigma$ is a characterisation of the noise over the transit timescale including systematic effects, and $S_{T}$ the detection threshold). For ground-based surveys, values of $\sigma=1-3 \times 10^{-3}$ are typical, but lower values are expected for space photometry.

Figure 9 shows the position of the candidates in the magnitude vs. transit signal $\left(d n^{1 / 2}\right)$ plane. We use the lower envelope of the candidates confirmed by the follow-up as being genuine transiting planets or eclipsing binaries to estimate the effective detection threshold. Rather surprisingly, the threshold shows no dependence on magnitude. This is the case when correlated fluctuations (due either to instrumental effects or to real variability in the stellar flux) dominate the detection statistic compared to random noise. The dashed line shows a limit at $d \cdot n^{1 / 2}=0.009$.

For comparison, the dotted line shows the "ideal" CoRoT detection limit, using photon-noise limited statistics and $S_{T}=10$ (such as used for instance in the pre-launch estimates of Bordé et al. 2003). The detection threshold is substantially higher than in pre-launch models, especially for the bright targets. The detection was carried out with a preliminary reduction pipeline and detrending algorithms that have been improved since, so that it is possible that some low-SNR candidates can be recovered at the brightest magnitudes in the future. In Fig. 9, one may see that 4 transiting candidates lie between both lines of expected and actual detection thresholds (E1-0783 and 3 candidates from Paper I). They were not followed-up as single 
transits or unpromising candidates, and are not considered further in discussing the threshold.

\section{Hot Jupiters}

Figure 9 shows that all transits with amplitude higher than $d n^{1 / 2}>3 \times 10^{-3}$ are detectable. Consequently, all transiting hot Jupiters should be detected, since with transit depths near $10^{-2}$ and $n \gg 1$, they will clearly exceed this limit. Previous surveys have established that the frequency of transiting hot Jupiters in a magnitude-limited galactic population is of the order of 1 per 2000 main-sequence stars (Gaudi et al. 2005; Fressin et al. 2007). There are about 8000 dwarfs among the IRa01 targets, so that 4 transiting hot Jupiters are expected, weighing towards the fainter end of the survey.

In this instance, 2 hot Jupiters were detected, around targets in the brighter end of the sample. The difference between the expectations and what was observed is not significant in itself, but the tendency of the number of detections to be lower than expectations and always situated at the brighter end of the sample is confirmed in all CoRoT fields analysed so far. The first six planets revealed by CoRoT all have host stars in the bright half of the target range, on average about two per field.

We see three possible explanations for the dearth of hot Jupiters detected thus far in CoRoT fields: (1) There is a real lack of Jupiter-like planets at the faint end of the sample; (2) Transiting hot Jupiters around fainter targets were rejected when selecting candidates from the detection list; and (3) Transiting hot Jupiters are included in the sample, but were not confirmed by radial velocity.

At this point, all these explanations seem unlikely:

1. the colour-magnitude diagram of the target population does not show any population change between the bright and faint end of the target range;

2. hot Jupiter transits are easy to identify at the level of signalto-noise afforded by the CoRoT data, and in this first Corot run, every effort was made to err on the side of caution when including candidates for follow-up;

3. the status of all but five planetary candidates (E1-4108, E1-4591, E1-4617, E2-4467 and E1-4998) was determined by complementary observations; we cannot conclude on the four last cases because their parent star has a rotationally broadened line profile.

Our best guess is that the reason is a combination of (2) and (3). The difficulty of identifying and confirming transiting planets in the CoRoT sample is a very steep function of magnitude. At the faintest end (as for E1-4617, E2-4467 and E1-4998), random noise and instrumental systematics can affect the detectability and apparent shape of the transit signal. On the radial-velocity side, the targets become too faint for follow-up with 1-2 m class telescopes (CORALIE and SOPHIE in our case), and require expensive 4-10-m class telescopes (HARPS in our case). Background eclipses also become much harder to rule out with ground-based photometry. It is probably not a coincidence that other surveys, such as TrES, also see their yields of hot Jupiter detections biased towards the bright end compared to statistical simulations.

Thus, when interpreting the present CoRoT results statistically, we warn that an effective cut at $R<14$ may be needed in the detection/confirmation model, beyond which the actual characteristics of the detection/confirmation procedure are very difficult to model.

\section{Small planets}

Close-in planets in the mass range between Neptune and Saturn are known from radial-velocity surveys to be rarer than hot Jupiters around normal stars. Therefore, we expect a rate of detection of the order of unity or less per CoRoT field, even if the candidate census is complete. The lack of detection in IRa01 is obviously compatible with this estimate, and does not allow further conclusions.

Smaller planets may be more abundant. A detection threshold near $d n^{1 / 2}=1.3 \times 10^{-2}$ corresponds for instance to a transit depth of $3 \times 10^{-3}$ with $P=3$ days, given the $\sim 60$-day duration of the run. This is the signal of a $4.5-R_{\text {Earth }}$ planet across a solartype star. Therefore, this set of candidates has a low sensitivity to planets smaller in size than Neptune. It could have been detected in IRa01 only on very close orbits or around small stars (which are rare in such a magnitude-limited sample).

\section{Conclusion}

Table 14 gives the state-of-the-art solution for all 29 IRa01 candidates for which follow-up observations are considered as complete. We unambiguously identify 1) two planets; 2) nine eclipsing and spectroscopic binaries; 3) five background eclipsing binaries located a few arcsec away from the main CoRoT target and included in the photometric mask; 4) five diluted eclipsing binaries or possible triple systems. The remaining candidates fall into two categories: 5) five candidates have a hot parent star for which no radial-velocity measurement is possible; and 6) three stars show no detectable radial-velocity variation over time. Over these eight unsolved cases, several have not been followed-up with ground-based photometry to check for a nearby background eclipsing binary, so this scenario cannot be excluded. Finally, a single candidate, E1-4108, is still a potential planetary system with confirmation feasible, although it would require a relatively large effort in gathering more data $(V=15.4$ and RV semi-amplitude less than $20 \mathrm{~m} / \mathrm{s}$ ).

The lessons learned from the IRa01 follow-up operations can be summarized as follows: 1) time-critical ground-based photometry is a key step in the confirmation process, and unfortunately, the main bottleneck of these operations. Such observations must be done not too early (one-two months after the discovery), but not too late (typically less than 2 years) to obtain precise ephemeris values and definite conclusions. In some configurations, transits with a good visibility are extremely rare; a good coverage in longitude is essential; 2) For faint targets, both photometry and radial-velocity becomes very difficult. Although for IRa01, several targets with magnitudes higher than 15 were actually followed up with HARPS, and identified, this requires a large effort and the collected data is not always conclusive. This is, however, where most of giant transiting planets would lie.

In order to be more efficient in future fields observed by CoRoT, one would need a stronger confidence in the lightcurve analysis components, since four candidates have had a late detection of their faint secondary. With refined tools, the CoRoT team can now use this information in a systematic way. Then, a more intensive effort on ground-based photometry is necessary, and as soon as possible after the transit detection. The identification of early-type stars and fast rotators may help screening the candidate list, but does not help resolve the scenarios. Finally, high-precision radial-velocity measurements on faint targets $(V>15)$ is time consuming with HARPS and did not 
show promising results for IRa01; such measurements were not given high priority for the follow-up operations, not allowing the confirmation of some giant planets.

\section{References}

Aigrain, S., Collier Cameron, A., Ollivier, M., et al. 2008, A\&A, 488, L43 Alonso, R., Alapini, A., Aigrain, S., et al. 2009, A\&A, 506, 353 Barge, P., Baglin, A., Auvergne, M., et al. 2008, A\&A, 482, L17 Bean, J. L. 2009, A\&A, 506, 369

Bordé, P., Rouan, D., \& Léger, A. 2003, A\&A, 405, 1137

Bouchy, F., Moutou, C., \& Queloz, D. 2009, IAU Symp., 253, 129
Brown, T. M. 2003, ApJ, 593, L125

Carpano, S., Cabrera, J., Alonso, R., et al. 2009, A\&A, 506, 491 Deleuil, M., Meunier, J.-C., Surace, C., et al. 2009, AJ, 138, 649

Fressin, F., Guillot, T., Morello, V., \& Pont, F. 2007, A\&A, 475, 729

Gaudi, B. S., Seager, S., \& Mallen-Ornelas, G. 2005, ApJ, 623, 472

Gillon, M., Magain, P., Chantry, V., et al. 2007, in Transiting Extrapolar Planets

Workshop, ed. C. Afonso, D. Weldrake, \& T. Henning, ASP Conf. Ser., 366, 113

Gillon, M., Demory, B.-O., Triaud, A. H. M. J., et al. 2009, A\&A, 506, 359

Moutou, C., Bruntt, H., Guillot, T., et al. 2008, A\&A, 488, L47

Pont, F., Zucker, S., \& Queloz, D. 2006, MNRAS, 373, 231

Robin, A. C., Reylé, C., Derrière, S., \& Picaud, S. 2004, A\&A, 416, 157

Snellen, I. A. G., de Mooij, E. J. W., \& Albrecht, S. 2009, Nature, 459, 543

Surace, C., Alonso, R., Barge, P., et al. 2008, in SPIE Conf. Ser., 7019

Pages 331 to 336 (Tables 2 to 13,15 to 17 and Figs. 4 to 7 ) are available in the electronic edition of the journal at http://www. aanda.org 


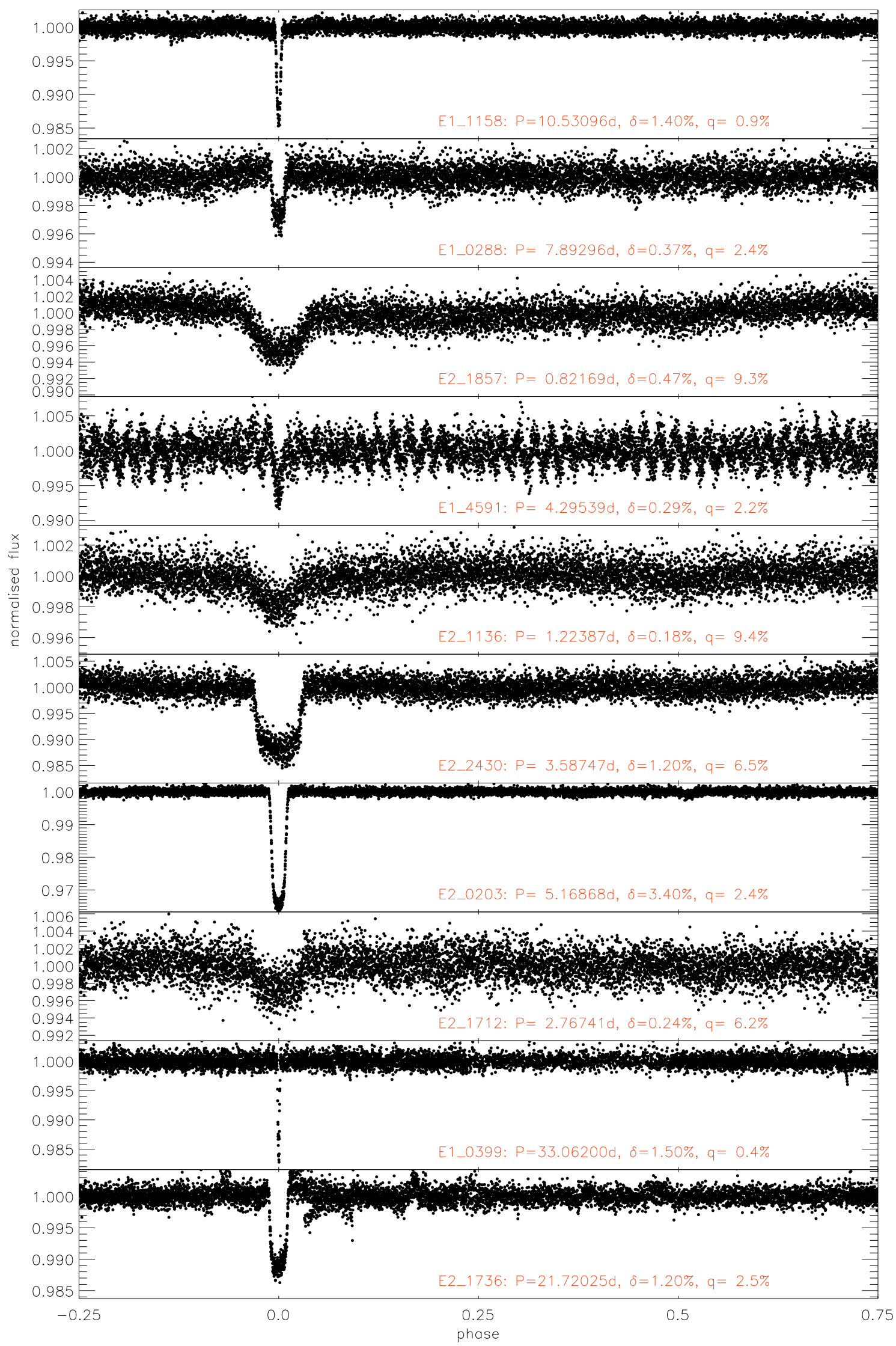

Fig. 4. CoRoT lightcurves of all candidates discussed in this paper: the light curve is phase-folded and the full orbital phase is shown. The iterative reconstruction filter (Alapini \& Aigrain, subm.) is applied to the data, in order to correct for long-term instrumental and activity-related features while preserving the variation at the transit period. 


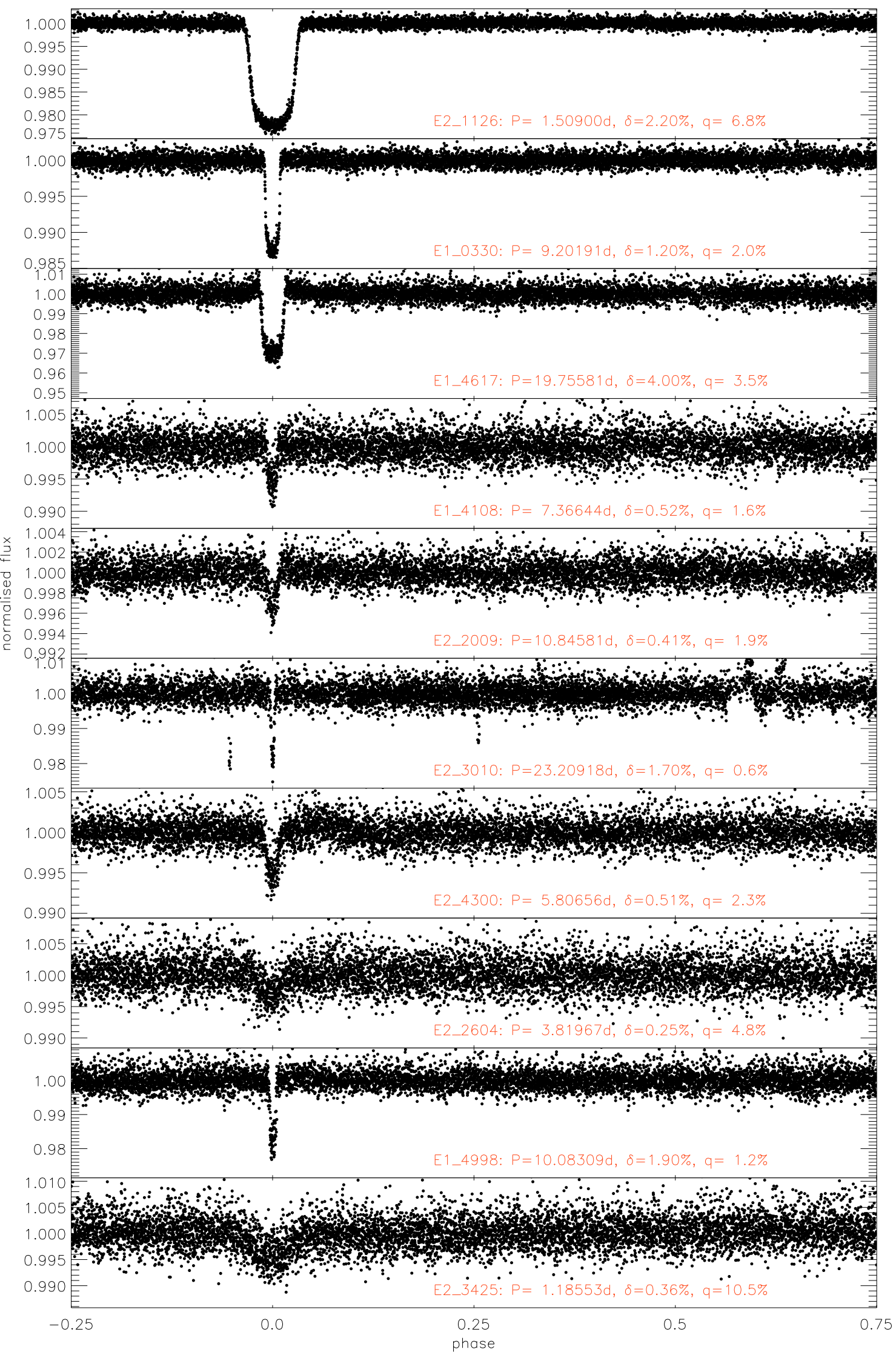

Fig. 5. CoRoT lightcurves of all candidates discussed in this paper (cont'd). 

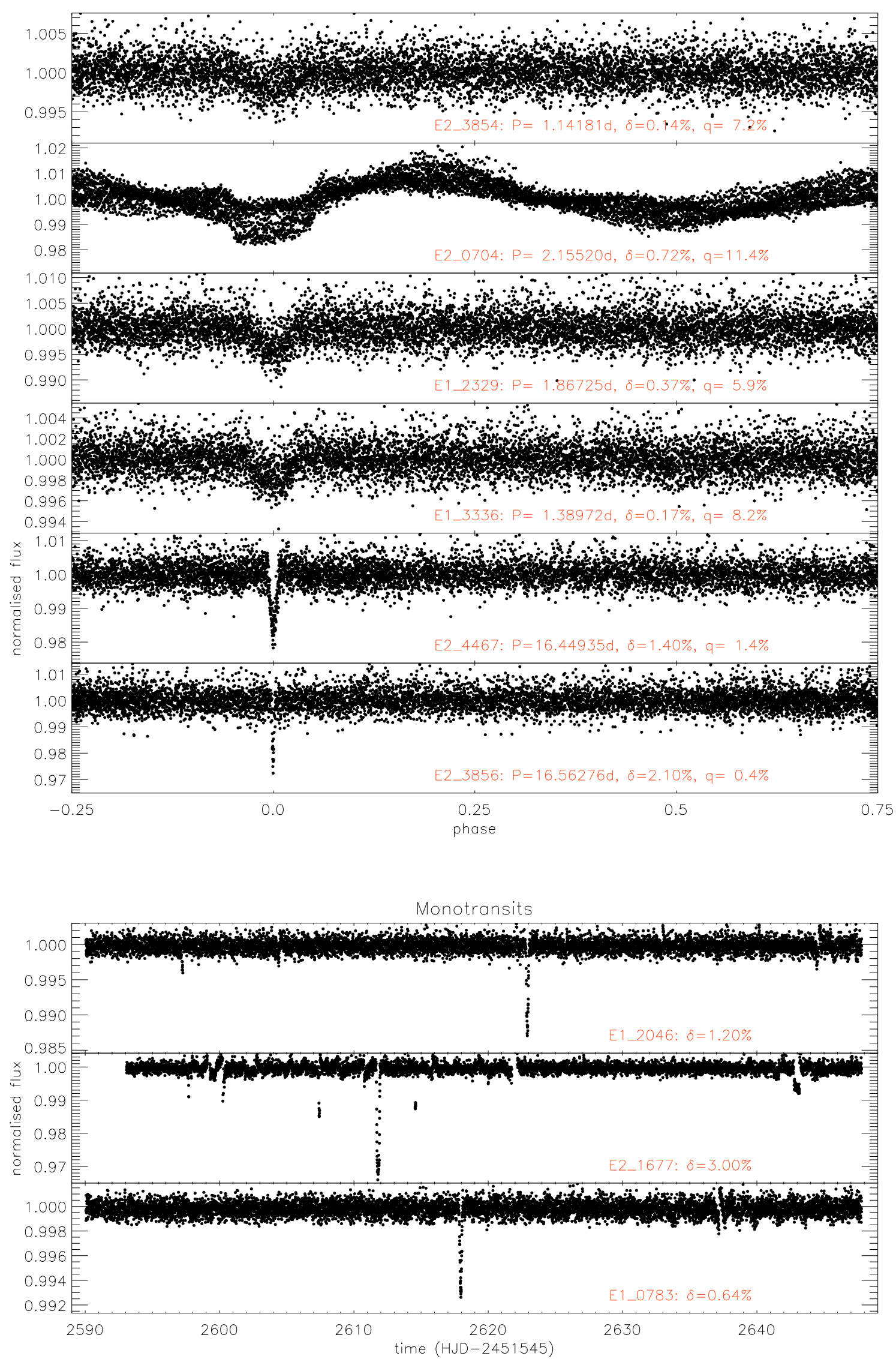

Fig. 6. CoRoT lightcurves of all candidates discussed in this paper (cont'd). For single transits, the full light curve is shown against Julian Date. 

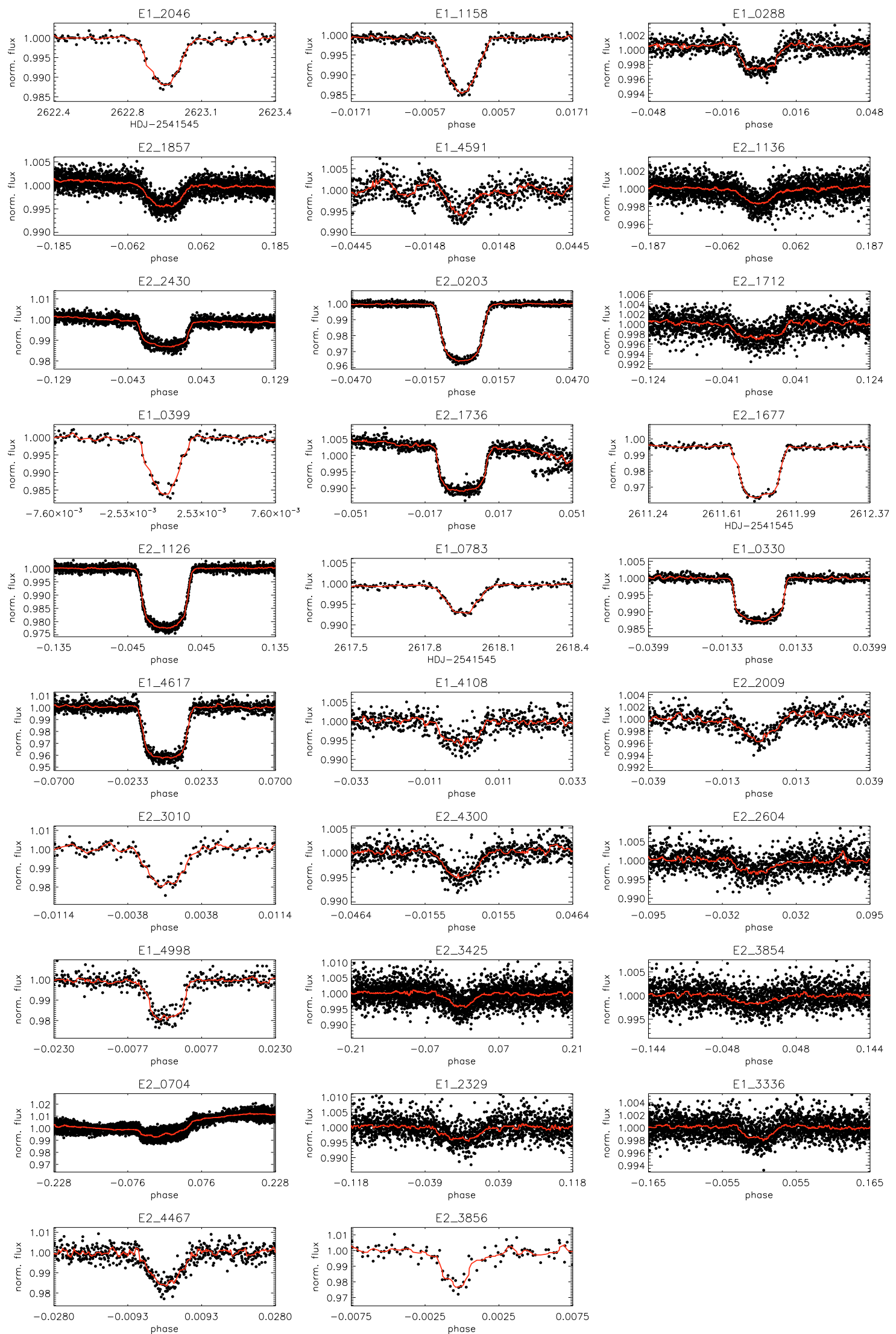

Fig. 7. CoRoT lightcurves of all candidates discussed in this paper (cont'd): zoom on the primary transits. The X-axis corresponds to the orbital phase when the period is determined, and Julian Date otherwise. 
Table 2. Pre-launch information on IRa01 transit candidates derived from multi-colour photometry.

\begin{tabular}{|c|c|c|c|c|c|c|c|c|c|c|c|c|}
\hline WinID & CoRoT ID & RA & Dec & $\overline{\overline{B^{a}}}$ & $V$ & $r$ & $\overline{J^{b}}$ & $\overline{K K}$ & SpT & $\bar{T}$ Teff $^{c}$ & Instr. & Conta $^{d}$ \\
\hline E1-2046 & 102723949 & $06: 4$ & 3.24 & 14.885 & 13.958 & 13.598 & 12.355 & 11.683 & K0III & 5000 & $\overline{\mathrm{PH}}$ & 0.0101 \\
\hline E2-3010 & 0106 & & $-02:$ & & 1 & & & & & 00 & $\mathrm{H}$ & 16 \\
\hline 54 & 1669 & & 1 & & & & & & K5III & & $\mathrm{PH}$ & 38 \\
\hline & 4464 & & & & & & & & $5 \mathrm{~V}$ & (10 & PF & 10 \\
\hline & 763847 & & 24 & 4 & 99 & 12 & 30 & 74 & F0V & 7250 & $\mathrm{PH}$ & 004 \\
\hline & 5481 & & 31 & & 13.308 & 68 & 58 & 81 & G1V & 5800 & $\mathrm{PH}$ & .000 \\
\hline 30 & 5260 & 78 & $-03:$ & 15.74 & 14.893 & 69 & 22 & 48 & $\mathrm{~F} 2 \mathrm{~V}$ & 7000 & SO & .019 \\
\hline-0399 & 829121 & .37 & $3: 11.62$ & 14.708 & 13.933 & 13.663 & 2.543 & 12.126 & G4V & 5550 & $\mathrm{PH}$ & .003 \\
\hline 1736 & 5534 & $6: 47: 30.58$ & $-02: 55: 04.12$ & 14.681 & 14.062 & 13.845 & 2.762 & 12.412 & G0V & 5900 & $\mathrm{PH}$ & 0.086 \\
\hline E2-2604 & 102805893 & 3.99 & $-02:($ & 16.513 & 15.703 & 15.416 & 4.110 & 3.635 & G8V & 5200 & $\mathrm{PH}$ & 0.0194 \\
\hline E2-1136 & 2809071 & 36 & $-03:($ & 97 & 13.228 & & 11 & 2.496 & A9V & 7400 & $\mathrm{PH}$ & 8 \\
\hline E2-4300 & 2430 & & $-02:$ & 234 & 52 & & & 2.817 & G3V & 5600 & FI & .212 \\
\hline & & & -00 & & & & 69 & & 1 & 6000 & 11 & \\
\hline & & & -02 & & & & & & F9V & 950 & $\mathrm{PH}$ & .006 \\
\hline & & & & & & & & & MOII & 3800 & $\mathrm{PH}$ & 0.039 \\
\hline & & & -0 & & & & & 15 & G9IV & 5100 & $\mathrm{PH}$ & 0.062 \\
\hline & & & 6 & & & & & & K8V & 4050 & $\mathrm{PH}$ & 0.016 \\
\hline & & & -0 & & & & & & G7V & 0 & $\mathrm{PH}$ & 0.002 \\
\hline & 57 & & $-01:$ & .246 & 52 & 20 & 51 & 60 & F2IV & 6800 & $\mathrm{PH}$ & 0.028 \\
\hline & 15 & & -02 & $1^{\prime}$ & 7 & 4 & 33 & 70 & K2III & 4800 & $\mathrm{P}$ & 0.000 \\
\hline-4591 & 806520 & 25 & -01 : & .452 & 3.863 & 13.697 & 05 & 2.418 & F9V & 6000 & $\mathrm{PH}$ & 0.315 \\
\hline 1712 & 6302 & 59 & $-03:($ & 58 & .197 & 13.976 & 12.843 & 2.530 & F5IV & 6700 & HA & 0.04 \\
\hline-3425 & 817 & 2 & -02 : & 71 & 15.903 & 98 & 4.320 & 13.862 & G7III & 5800 & HA & 0.26 \\
\hline E1-4998 & 1773 & 92 & $-01:$ & .41 & 15.71 & 75 & 14.354 & 3.909 & $\mathrm{G} 2 \mathrm{~V}$ & 5700 & $\mathrm{PH}$ & .258 \\
\hline E2-0704 & 5472 & 0 & -02 & J & 5 & 55 & 14 & 12. & G7III & 5400 & HA & 0.102 \\
\hline E2-1677 & 874481 & 2 & -02 & 3 & 41 & 39 & 12.989 & 12.707 & F4V & 6500 & $\mathrm{PH}$ & 0.015 \\
\hline 108 & 9966 & & 6 & 2 & 2 & 36 & 71 & 12.880 & $\mathrm{~K} 2 \mathrm{~V}$ & 5100 & HA & 0.0437 \\
\hline E2-1126 & 102890318 & 9.20 & $-03:$ & 14.197 & 13.624 & 13.431 & 12.462 & 12.149 & G0V & 5950 & UV & 0.0383 \\
\hline E1-0330 & 102912369 & $06: 48: 46.70$ & $-00: 40: 21.97$ & 14.312 & 13.694 & 13.450 & 12.619 & 12.288 & F0V & 6190 & UV & 0.003 \\
\hline
\end{tabular}

${ }^{a}$ Optical magnitudes were measured at the Isaac Newton Telescope with the Wide Field Camera; $B$ and $V$ filters are from the Harris system, and $r$ from the Sloan system. ${ }^{b} J$ and $K$ magnitudes are 2MASS measurements. ${ }^{c}$ The origin of the estimate for effective temperature (Teff), spectral type and luminosity class (SpT) may be from spectral analysis (HA for HARPS, UV for UVES, SO for SOPHIE) or by default, from multicolour photometry. "conta" is the ratio between the background flux and the total flux.

Table 3. Radial-velocity measurements of E2-3854. SO stands for SOPHIE and HA for HARPS.

\begin{tabular}{llllll}
\hline \hline JD-2 400 000. & $\phi$ & $\begin{array}{l}\text { RV1 } \\
{\left[\mathrm{km} \mathrm{s}^{-1}\right]}\end{array}$ & $\begin{array}{l}\text { RV2 } \\
{\left[\mathrm{km} \mathrm{s}^{-1}\right]}\end{array}$ & $\begin{array}{l}\sigma \\
{\left[\mathrm{km} \mathrm{s}^{-1}\right]}\end{array}$ & Instr. \\
\hline 54494.416 & 0.36 & 1.56 & 98.35 & 0.2 & SO \\
54505.364 & 0.95 & 80.8 & 12.55 & 0.5 & SO \\
\hline
\end{tabular}

Table 4. Radial-velocity measurements of 0102763847/E1-1158.

\begin{tabular}{lllll}
\hline \hline JD-2 400 000. & $\phi$ & $\begin{array}{l}\text { RV } \\
{\left[\mathrm{km} \mathrm{s}^{-1}\right]}\end{array}$ & $\begin{array}{l}\sigma \\
{\left[\mathrm{km} \mathrm{s}^{-1}\right]}\end{array}$ & Instr. \\
\hline 54521.328 & 0.21 & -17.795 & 0.070 & SO \\
54526.291 & 0.69 & 28.152 & 0.080 & SO \\
\hline
\end{tabular}

Table 5. Radial-velocity measurements of E1-0399. CO stands for CORALIE at the Euler $120 \mathrm{~cm}$ Swiss telescope.

\begin{tabular}{lllll}
\hline \hline JD-2 400 000. & $\phi$ & $\begin{array}{l}\text { RV } \\
{\left[\mathrm{km} \mathrm{s}^{-1}\right]}\end{array}$ & $\begin{array}{l}\sigma \\
{\left[\mathrm{km} \mathrm{s}^{-1}\right]}\end{array}$ & Instr. \\
\hline 54477.734 & 0.86 & 43.05 & 0.08 & $\mathrm{CO}$ \\
54495.566 & 0.39 & 15.43 & 0.11 & $\mathrm{CO}$ \\
\hline
\end{tabular}

Table 6. Radial-velocity measurements of E2-3856.

\begin{tabular}{lllll}
\hline \hline JD-2 400 000. & $\phi$ & $\begin{array}{l}\text { RV } \\
{\left[\mathrm{km} \mathrm{s}^{-1}\right]}\end{array}$ & $\begin{array}{l}\sigma \\
{\left[\mathrm{km} \mathrm{s}^{-1}\right]}\end{array}$ & Instr. \\
\hline 54530.641 & 0.24 & 4.74 & 0.02 & SO \\
54549.546 & 0.39 & 26.19 & 0.02 & SO \\
\hline
\end{tabular}

Table 7. Radial-velocity measurements of E2-2430.

\begin{tabular}{lllll}
\hline \hline JD-2 400 000. & $\phi$ & $\begin{array}{l}\text { RV } \\
{\left[\mathrm{km} \mathrm{s}^{-1}\right]}\end{array}$ & $\begin{array}{l}\sigma \\
{\left[\mathrm{km} \mathrm{s}^{-1}\right]}\end{array}$ & Instr. \\
\hline 54502.409 & 0.23 & 51.36 & 0.111 & $\mathrm{SO}$ \\
54504.401 & 0.79 & 97.25 & 0.098 & $\mathrm{SO}$ \\
\hline
\end{tabular}

Table 8. Radial-velocity measurements of 0102855534/E1-1736.

\begin{tabular}{lllll}
\hline \hline JD-2 400 000. & $\phi$ & $\begin{array}{l}\text { RV } \\
{\left[\mathrm{km} \mathrm{s}^{-1}\right]}\end{array}$ & $\begin{array}{l}\sigma \\
{\left[\mathrm{km} \mathrm{s}^{-1}\right]}\end{array}$ & Instr. \\
\hline 54497.471 & 0.29 & 27.07 & 0.121 & SO \\
54507.424 & 0.75 & 57.55 & 0.099 & SO \\
\hline
\end{tabular}


Table 9. Radial-velocity measurements of 0102855472/E2-0704.

\begin{tabular}{lllll}
\hline \hline JD-2 400 000. & $\phi$ & RV & $\sigma$ & Instr. \\
& CoRoT & {$\left[\mathrm{km} \mathrm{s}^{-1}\right]$} & $\begin{array}{l}\left.\sigma \mathrm{km} \mathrm{s}^{-1}\right] \\
\end{array}$ \\
\hline 54526.599 & 0.78 & 39.36 & 0.031 & HA \\
54527.611 & 0.24 & 39.32 & 0.032 & HA \\
\hline
\end{tabular}

Table 10. Radial-velocity measurements of 0102798247/E2-1857.

\begin{tabular}{llllll}
\hline \hline JD-2 400 000. & $\phi$ & $\phi$ & RV & $\sigma$ & Instr. \\
& CoRoT & RV & {$\left[\mathrm{km} \mathrm{s}^{-1}\right]$} & $\begin{array}{l}\sigma \\
{\left[\mathrm{km} \mathrm{s}^{-1}\right]}\end{array}$ \\
\hline 54499.329 & 0.353 & 0.437 & 16.35757 & 0.0724 & SO \\
54501.369 & 0.836 & 0.670 & 25.33129 & 0.0546 & SO \\
54514.361 & 0.647 & 0.158 & 16.97243 & 0.0681 & SO \\
54515.306 & 0.797 & 0.266 & 14.94759 & 0.0634 & SO \\
54518.399 & 0.561 & 0.620 & 23.51338 & 0.0403 & SO \\
54525.281 & 0.937 & 0.408 & 15.583 & 0.064 & SO \\
54527.305 & 0.400 & 0.640 & 22.97 & 0.05 & SO \\
54528.326 & 0.643 & 0.757 & 27.058 & 0.050 & SO \\
54548.542 & 0.246 & 0.072 & 20.746 & 0.070 & HA \\
54549.506 & 0.419 & 0.182 & 16.310 & 0.079 & HA \\
54550.540 & 0.678 & 0.300 & 14.424 & 0.111 & HA \\
54551.549 & 0.905 & 0.416 & 16.000 & 0.055 & HA \\
\hline
\end{tabular}

Table 11. Radial-velocity measurements of 0102788073/E2-2009.

\begin{tabular}{llllll}
\hline \hline JD-2 400 000. & $\phi$ & $\phi$ & RV & $\sigma$ & Instr. \\
& CoRoT & 2P & {$\left[\mathrm{km} \mathrm{s}^{-1}\right]$} & {$\left[\mathrm{km} \mathrm{s}^{-1}\right]$} & \\
\hline 54503.426 & 0.344 & 0.672 & 79.90 & 0.054 & SO \\
54519.398 & 0.817 & 0.408 & 80.00 & 0.025 & SO \\
54527.351 & 0.550 & 0.775 & 79.95 & 0.058 & SO \\
54528.365 & 0.644 & 0.822 & 79.96 & 0.041 & SO \\
54540.348 & 0.749 & 0.374 & 79.83 & 0.040 & SO \\
54545.346 & 0.209 & 0.605 & 79.87 & 0.020 & SO \\
54546.329 & 0.300 & 0.650 & 79.36 & 0.030 & SO \\
54548.501 & 0.50 & 0.75 & $79.94(\mathrm{~F} 0)$ & 0.047 & HA \\
54548.501 & 0.50 & 0.75 & $80.078(\mathrm{G} 2)$ & 0.047 & HA \\
54548.501 & 0.50 & 0.75 & $80.131(\mathrm{~K} 5)$ & 0.047 & HA \\
54551.515 & 0.77 & 0.89 & $79.987(\mathrm{~F} 0)$ & 0.035 & HA \\
54551.515 & 0.77 & 0.89 & $79.928(\mathrm{G} 2)$ & 0.035 & HA \\
54551.515 & 0.77 & 0.89 & $79.915(\mathrm{~K} 5)$ & 0.035 & HA \\
\hline
\end{tabular}

Table 12. Radial-velocity measurements of 0102869286/E1-2329.

\begin{tabular}{llllll}
\hline \hline JD-2 400 000. & $\phi$ & $\phi$ & RV & $\sigma$ & Instr. \\
& CoRoT & 2P & {$\left[\mathrm{km} \mathrm{s}^{-1}\right]$} & {$\left[\mathrm{km} \mathrm{s}^{-1}\right]$} & \\
\hline 54519.638 & 0.693 & 0.847 & 15.786 & 0.02 & HA \\
54522.609 & 0.284 & 0.642 & 15.822 & 0.017 & HA \\
54525.556 & 0.863 & 0.431 & 15.743 & 0.014 & HA \\
\hline
\end{tabular}

Table 13. Radial-velocity measurements of 0102876631/E1-3336.

\begin{tabular}{lllll}
\hline \hline JD-2 400 000. & $\phi$ & $\begin{array}{l}\text { RV } \\
{\left[\mathrm{km} \mathrm{s}^{-1}\right]}\end{array}$ & $\begin{array}{l}\sigma \\
{\left[\mathrm{km} \mathrm{s}^{-1}\right]}\end{array}$ & Instr. \\
\hline 54497.425 & 0.163 & 71.425 & 0.036 & SO \\
54502.360 & 0.715 & 71.583 & 0.033 & SO \\
54503.376 & 0.446 & 71.466 & 0.047 & SO \\
54517.626 & 0.700 & $71.62(\mathrm{~F} 0)$ & 0.021 & HA \\
& & $71.595(\mathrm{G} 2)$ & & \\
& & $71.606(\mathrm{~K} 5)$ & & \\
54519.547 & 0.082 & $71.397(\mathrm{~F} 0)$ & 0.019 & HA \\
& & $71.463(\mathrm{G} 2)$ & & \\
54522.552 & 0.245 & $71.492(\mathrm{~K} 5)$ & & HA \\
& & $71.478(\mathrm{~F} 0)$ & 0.011 & \\
& & $71.546(\mathrm{G} 2)$ & & \\
\hline
\end{tabular}

Table 15. Radial-velocity measurements of E1-4998.

\begin{tabular}{lllll}
\hline \hline JD-2 400 000. & $\phi$ & $\begin{array}{l}\text { RV } \\
{\left[\mathrm{km} \mathrm{s}^{-1}\right]}\end{array}$ & $\begin{array}{l}\sigma \\
{\left[\mathrm{km} \mathrm{s}^{-1}\right]}\end{array}$ & Instr. \\
\hline 54487.645 & 0.24 & 25. & 0.2 & HA \\
54524.625 & 0.91 & 3. & 0.4 & HA \\
\hline
\end{tabular}

Table 16. Radial-velocity measurements of E2-3425.

\begin{tabular}{lllll}
\hline \hline JD-2 400 000. & $\phi$ & $\begin{array}{l}\text { RV } \\
{\left[\mathrm{km} \mathrm{s}^{-1}\right]}\end{array}$ & $\begin{array}{l}\sigma \\
{\left[\mathrm{km} \mathrm{s}^{-1}\right]}\end{array}$ & Instr. \\
\hline 54744.875 & 0.74 & 27.6847 & 0.028 & HA \\
54746.863 & 0.41 & 27.7201 & 0.036 & HA \\
\hline
\end{tabular}

Table 17. Radial-velocity measurements of E1-4108.

\begin{tabular}{lllll}
\hline \hline JD-2 400 000. & $\phi$ & $\begin{array}{l}\text { RV } \\
{\left[\mathrm{km} \mathrm{s}^{-1}\right]}\end{array}$ & $\begin{array}{l}\sigma \\
{\left[\mathrm{km} \mathrm{s}^{-1}\right]}\end{array}$ & Instr. \\
\hline 54516.602 & 0.39 & 99.15 & 0.015 & HA \\
54518.645 & 0.66 & 99.14 & 0.019 & HA \\
54519.592 & 0.80 & 99.15 & 0.018 & HA \\
\hline
\end{tabular}

